\title{
Controllable Non-specific Protein Adsorption by Charged Hyperbranched Polyglycerol Thin Films
}

\author{
Yaming Yu ${ }^{a, b^{*}}$, Holger Frey $b^{b^{*}}$
}

a College of Materials Science and Engineering, Huaqiao University, Xiamen 361021, China

b Institute of Organic Chemistry, Johannes Gutenberg-University Mainz, Duesbergweg 10-14, D-55128 Mainz, Germany 


\section{Synthesis of Anionic Hyperbranched Polyglycerols (Anionic hbPGs)}

Anionic hbPG with carboxylic acid groups was obtained by succinate functionalization of hbPG according to a previously published strategy. ${ }^{1}$ Hyperbranched polyglycerol (DP=39 (degree of polymerization), $2.31 \mathrm{~g}, 0.032 \mathrm{~mol})$ was treated with succinic anhydride $(1.92 \mathrm{~g}$, $0.019 \mathrm{~mol}$ ) at $40^{\circ} \mathrm{C}$ in the presence of triethylamine (TEA, $0.1 \mathrm{ml}$ ), in anhydrous N,N-dimethylformamide (DMF; $20 \mathrm{ml}$ ) (shown in Scheme S1). After evaporation of the solvent under vacuum line, the residue was dissolved in distilled water, dialyzed by employing a 1200 cut-off membrane, and dried in vacuum oven at $40^{\circ} \mathrm{C}$ for 2 days as a colorless paste (yield 76\%). Anionic $\mathrm{hbPG}_{\mathrm{m}}\left(\mathrm{COO}^{-} \mathrm{H}^{+}\right)_{\mathrm{n}}$ with different molecular weights were synthesized, where $\mathrm{m}$ is the degree of polymerization $\left(\mathrm{DP}_{\mathrm{n}}\right)$ plus three, and $\mathrm{n}$ is the conversion of $-\mathrm{OH}$ groups into $-\mathrm{COOH}$ groups. They are $\mathrm{hbPG}_{42}\left(\mathrm{COO}^{-} \mathrm{H}^{+}\right)_{28}$ and $\mathrm{hbPG}_{90}\left(\mathrm{COO}^{-} \mathrm{H}^{+}\right)_{52}$ respectively. Bovine serum albumin and fibrinogen were purchased from Sigma-Aldrich and used without further purification. Other chemicals mentioned in this work were all purchased from Sigma-Aldrich.
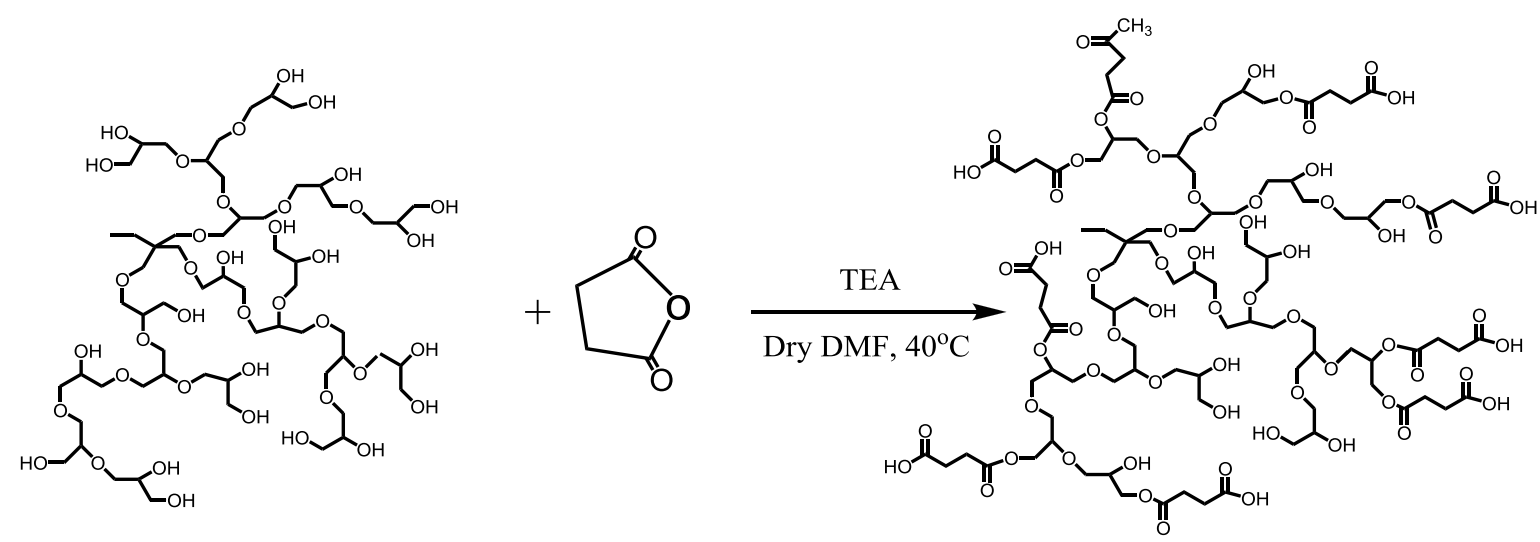

Scheme S1. Succinate functionalization of hyperbranched polyglycerols into anionic hyperbranched polyglycerols with carboxylic acid groups.

${ }^{1} \mathrm{H}$ NMR $\left(\mathrm{D}_{2} \mathrm{O}, 300 \mathrm{MHz}\right)$ for $\mathrm{hbPG}_{42}\left(\mathrm{COO}^{-} \mathrm{H}^{+}\right)_{28}: \delta=5.14 \mathrm{ppm}(\mathrm{br}, \mathrm{s} ; \mathrm{CHOCO}, 1 \mathrm{H})$, 4.26-3.88 ppm (broad m, $\mathbf{C H}_{\mathbf{2}} \mathrm{OCO}, 2 \mathrm{H}$ ), 3.61-3.49 ppm (br, m, hb PG-H), 2.59 ppm (br, s, OCOCH $\mathbf{C}_{2} \mathrm{CH}_{2} \mathrm{COOH}, 4 \mathrm{H}$ ), 1.27 ppm (broad s, $\left.\mathrm{CH}_{3} \mathrm{CH}_{2} \mathrm{C}\left(\mathrm{CH}_{2} \mathrm{O}\right)_{3}, 2 \mathrm{H}\right), 0.75$ ppm (broad s, $\left.\mathrm{CH}_{3} \mathrm{CH}_{2} \mathrm{C}\left(\mathrm{CH}_{2} \mathrm{O}\right)_{3}, 3 \mathrm{H}\right)$.

${ }^{1} \mathrm{H}$ NMR $\left(\mathrm{D}_{2} \mathrm{O}, 300 \mathrm{MHz}\right)$ for $\mathrm{hbPG}_{90}\left(\mathrm{COO}^{-} \mathrm{H}^{+}\right)_{52}: \delta=4.99 \mathrm{ppm}(\mathrm{br}, \mathrm{s} ; \mathrm{CHOCO}, 1 \mathrm{H})$, 4.12-3.91 ppm (broad m, $\mathrm{CH}_{2} \mathrm{OCO}, 2 \mathrm{H}$ ), 3.62-3.52 ppm (br, m, hb PG-H), 2.58 ppm (br, s, 
OCOCH $\left.\mathbf{H}_{2} \mathrm{CH}_{2} \mathrm{COOH}, 4 \mathrm{H}\right), 0.77$ ppm (broad s, $\left.\mathrm{CH}_{3} \mathrm{CH}_{2} \mathrm{C}\left(\mathrm{CH}_{2} \mathrm{O}\right)_{3}, 3 \mathrm{H}\right)$.
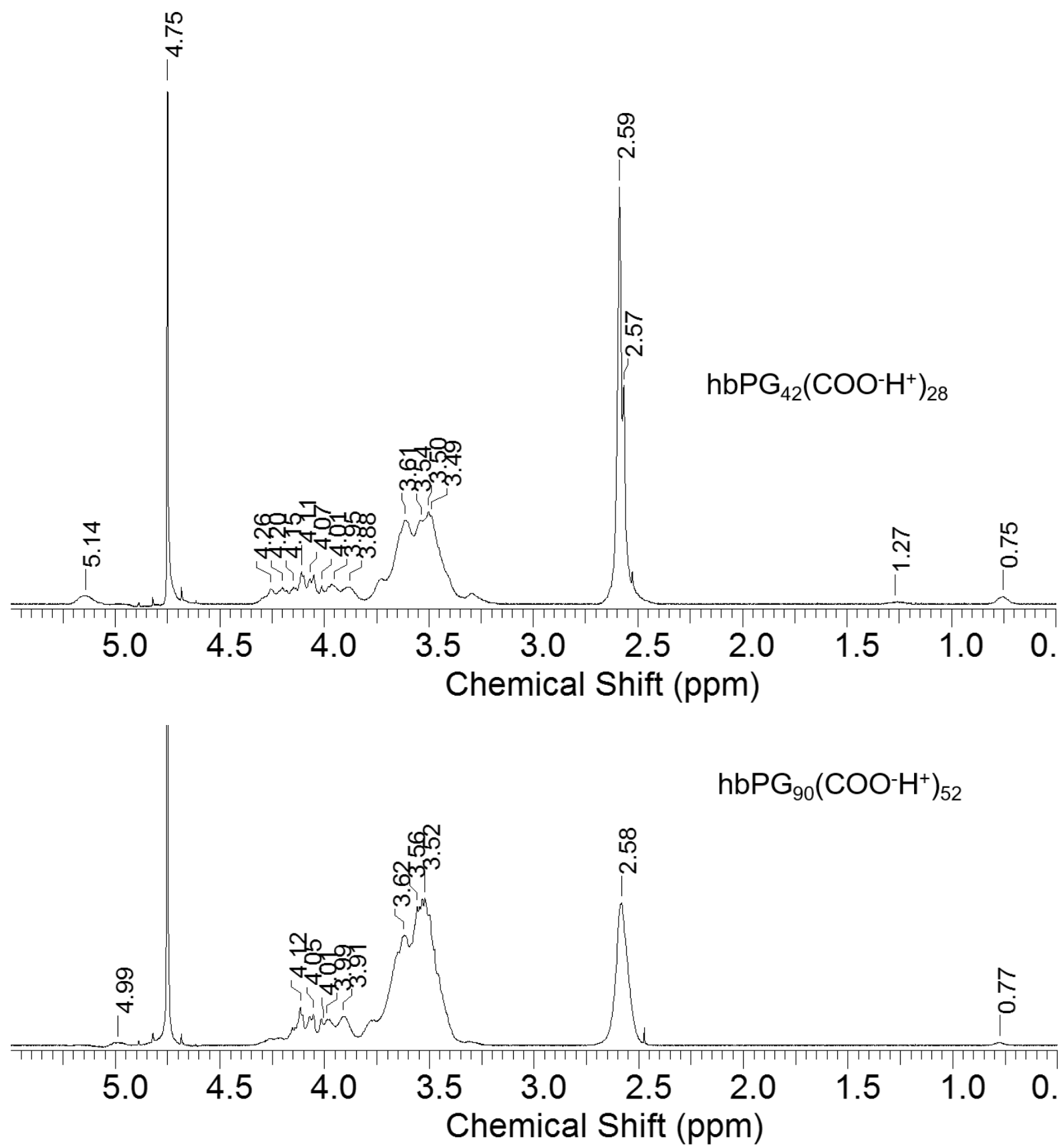

Figure S1. ${ }^{1} \mathrm{H}$ NMR of the modified hbPG into anionic hbPG with carboxylic acid groups.

\section{Synthesis of Cationic Hyperbranched Polyglycerol}

hbPG-Br: hbPG(Mw: 3000, DP $\left.{ }_{\mathrm{n}}: 39\right)$ (3 g, $\left.0.042 \mathrm{~mol}\right)$ and triphenophosphine (15 g , 0.06 mol) were dissolved in dry dimethylformamide (DMF) $(60 \mathrm{ml})$ and cooled to $0{ }^{\circ} \mathrm{C} .9 \mathrm{~g}(0.05$ 
mol) N-bromosuccinimide (NBS) was carefully added in small portions, and the reaction mixture was stirred at room temperature for $12 \mathrm{~h}$. DMF was removed under reduced pressure at $40{ }^{\circ} \mathrm{C}$ for 5 days. The residue was dialyzed by employing a 1200 cut-off membrane, and evacuated to afford compound $\mathrm{hbPG}-\mathrm{Br}$ as a brown paste. The reaction yield is $21 \%$.

${ }^{1} \mathrm{H}-\mathrm{NMR}\left(300 \mathrm{MHz}, \mathrm{CD}_{3} \mathrm{OD}\right): \delta=0.94$ ppm (br, s, $\left.\mathrm{CH}_{3} \mathrm{CH}_{2} \mathrm{C}\left(\mathrm{CH}_{2} \mathrm{O}\right)_{3}-\mathrm{hbPG}-\mathrm{Br}, 3 \mathrm{H}\right), 1.46$ ppm (br, s, $\mathrm{CH}_{3} \mathrm{CH}_{2} \mathrm{C}\left(\mathrm{CH}_{2} \mathrm{O}\right)_{3}$-hbPG-Br, 2H), 2.98 ppm (br, s, hbPG-CH$\left.H_{2}-\mathrm{Br}, 2 \mathrm{H}\right), 3.64-3.95$ ppm (br, m, hbPG backbone), 4.19-4.46 ppm (br, m, hbPG-CH-Br, H).

hbPG-N ${ }^{+}\left(\mathrm{CH}_{3}\right)_{3} \mathrm{Br}^{-}$(cationic hbPG): hbPG-Br (1.1 g, $0.07 \mathrm{mmol}$ ) was dissolved in distilled tetrahydrofuran (THF) $(200 \mathrm{~mL})$ at room temperature and the solution was cooled to $-10{ }^{\circ} \mathrm{C}$. Condensed trimethylamine (TMA) $(20 \mathrm{ml})$ was dropwise added and the solution was stirred for $24 \mathrm{~h}$ while gradually warming to room temperature. Finally, the solvents were removed under reduced pressure as a slight brown paste (yield: $31 \%$ ). Conversion yield of $-\mathrm{N}^{+}\left(\mathrm{CH}_{3}\right)_{3} \mathrm{Br}^{-}$from $-\mathrm{OH}$ over two steps is $12.4 \%$.

Yields of above product were calculated after extensive dialysis.

\footnotetext{
${ }^{1} \mathrm{H}$ NMR (300 MHz, D 20$)$ :

$\delta=0.87$ ppm (br, s, $\left.\mathrm{CH}_{3} \mathrm{CH}_{2} \mathrm{C}\left(\mathrm{CH}_{2} \mathrm{O}\right)_{3}-\mathrm{hbPG}_{-} \mathrm{N}^{+}\left(\mathrm{CH}_{3}\right)_{3} \mathrm{Br}^{-}, 3 \mathrm{H}\right), 2.56-2.94$ ppm (br, m, $\left.-\mathrm{N}^{+}\left(\mathrm{CH}_{3}\right)_{3} \mathrm{Br}{ }^{-}, 9 \mathrm{H}\right)$, 3.22-3.33 ppm (br, m, hbPG-CH $\left.\mathrm{H}_{2}-\mathrm{N}^{+}\left(\mathrm{CH}_{3}\right)_{3} \mathrm{Br}^{-}, 2 \mathrm{H}\right), 3.51-3.97$ ppm (br, m, hbPG backbone), 4.22-4.40 ppm (br, m, hbPG-CH-N $\left.\left(\mathrm{CH}_{3}\right)_{3} \mathrm{Br}^{-}, \mathrm{H}\right)$.
} 

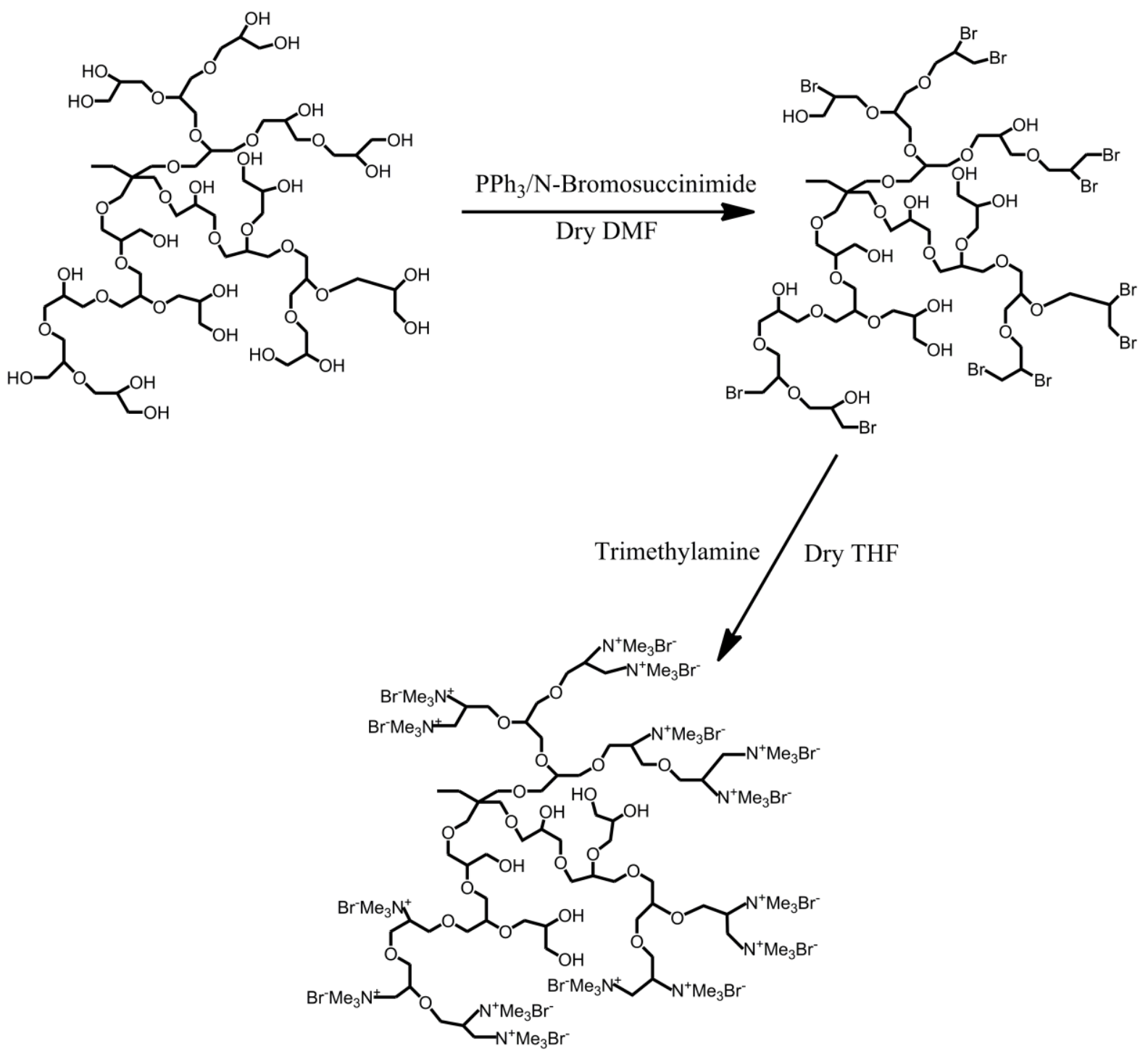

Scheme S2. Schematic illustration of synthesis steps for the cationic hyperbranched polyglycerol from the hyperbranched polyglycerol. 

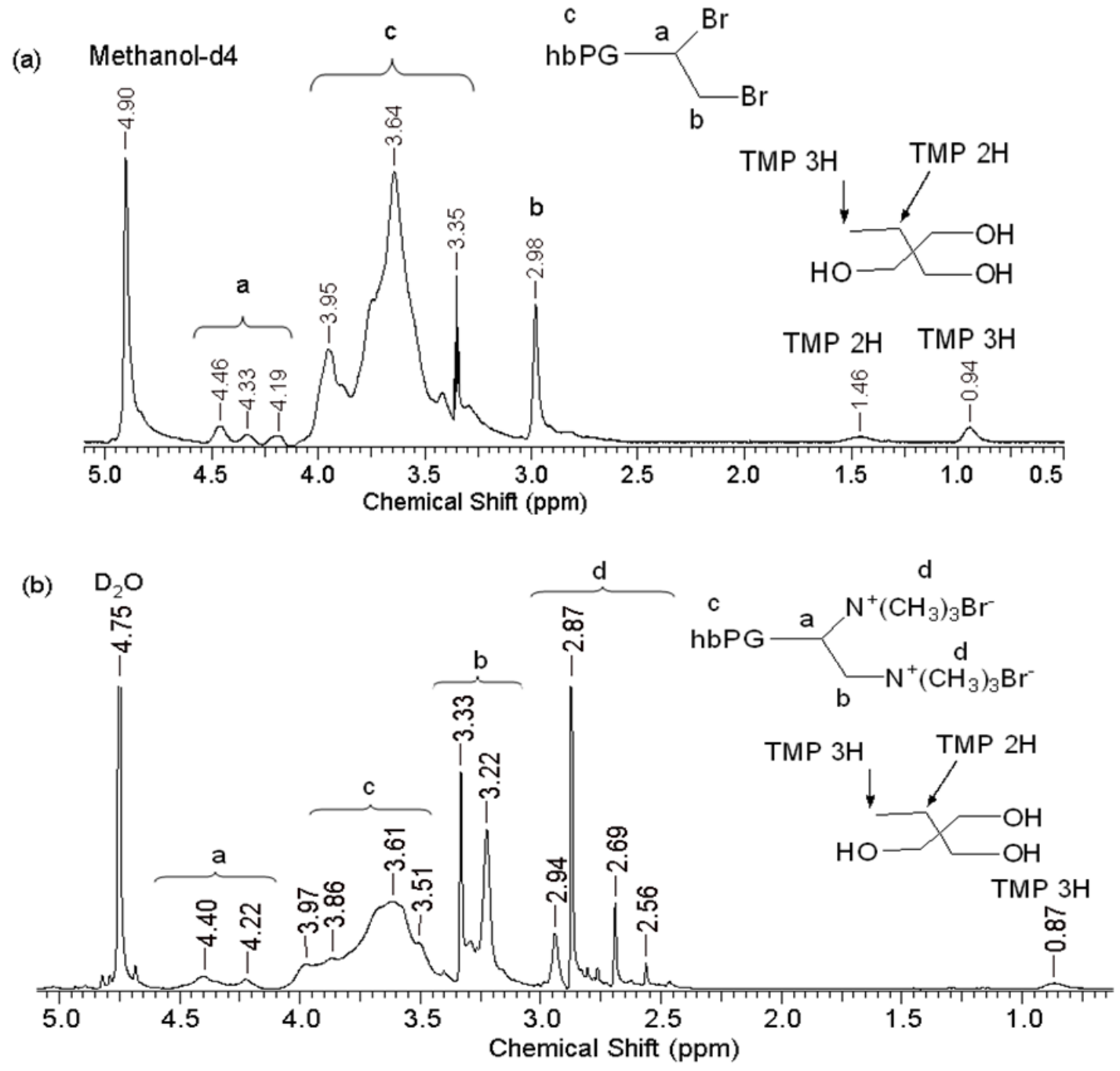

Figure S2. ${ }^{1} \mathrm{H}-\mathrm{NMR}$ spectra of the hbPG-Br (a) and hbPG-N ${ }^{+}\left(\mathrm{CH}_{3}\right)_{3} \mathrm{Br}^{-}$(b).

\section{Reference}

(1) Tziveleka, L. A.; Kontoyianni, C.; Sideratou, Z.; Tsiourvas, D.; Paleos, C. M. Novel Functional Hyperbranched Polyether Polyols as Prospective Drug Delivery Systems. Macromolecular Bioscience 2006, 6 (2), 161-169. 